\title{
Body integrity identity disorder: clinical features and ethical dimensions
}

\author{
Emma Barrow \& Femi Oyebode
}

\begin{abstract}
SUMMARY
Body integrity identity disorder (BIID) is a rare and complex identity disorder described by the desire to acquire a physical disability and an associated sense of incompleteness at being able-bodied. Individuals with the disorder often delay presentation until later in life because of perceived stigma about wishing to acquire a physical disability, and may have sought amputation already through 'underground' means or self-harm (attempts at self-amputation). In this article we present an account of the recent history and origins of the disorder, from its early descriptions and case reports through to the current neuropsychiatric theory of right superior parietal lobe dysfunction as basis for the disorder. We consider the epidemiology, pathogenesis and clinical features of this identity disorder of bodily integrity, highlighting the associations with conditions such as gender identity disorder. With this we then discuss the ethical considerations for available treatment options, mainly elective surgical amputation.
\end{abstract}

\section{LEARNING OBJECTIVES}

- Understand the current definition and clinical features of body integrity identity disorder

- Be familiar with the conceptual history of the disorder, epidemiology and current neuropsychiatric perspective

- Be aware of the ethical aspects of elective surgical amputation as a treatment for the disorder

\section{DECLARATION OF INTEREST}

None.

\section{KEYWORDS}

Ethics; self-harm; body integrity identity disorder.

The term body integrity identity disorder (BIID) refers to a psychiatric disorder characterised by the persistent desire to acquire a physical disability such as amputation or paraplegia, or other severe disability such as blindness. Individuals with BIID typically report a desire to achieve their sense of 'true-self' and that obtaining the desired amputation or disability would enable them to feel 'whole' or 'complete'. Although previously described in the literature as a paraphilia, there is a more recent and growing body of evidence to support a multifactorial psychiatric and neurological explanation for this disorder, with right superior parietal lobe dysfunction as one proposed neurobiological hypothesis. There are, similarly, strong parallels between BIID and other identity disorders, such as transsexualism and gender identity disorder. In BIID there is a mismatch between actual and perceived body schema, such that to have the desired amputation or acquired disability becomes a key part of the person's own identity. Onset of this desire also typically occurs in childhood or adolescence and is associated with chronic feelings of dysphoria which are somewhat alleviated by the desire to seek surgical intervention or to pretend to have the acquired disability - as with cross-dressing in transsexualism. Furthermore, several studies have shown that people with BIID sometimes go to extreme lengths to achieve the desired physical disability and therefore requests to surgeons for amputation of healthy limbs must balance the ethical argument opposing such a decision against the potential risk of self-harm.

The nosological status of this condition is yet to be fully determined. It is included in ICD-11 (WHO 2018) as body integrity dysphoria, code 6c21 but not included in DSM-V (APA 2013) except in section III for research purpose as body integrity disorder.

In this article we describe the historical development of the concept of BIID, consider the main features and the growing body of literature exploring the pathogenesis of the condition and address ethical aspects of the surgical management of the condition. It is important to make clear at the outset that the literature is relatively poor and much is dependent on self-reports from telephone interviews or online message boards. These sources limit the degree to which the findings can be regarded as secure and reliable. Nonetheless, given the paucity of information there is little choice but to rely on these reports, though taking account of the limitations.
Emma Barrow is a consultant liaison psychiatrist in general adult psychiatry working in the rapid assessment, interface and discharge (RAID) team at Birmingham Heartlands Hospital, Birmingham and Solihull Mental Health NHS Foundation Trust. Femi Oyebode is a consultant psychiatrist at The Barberry, which is part of the National Centre for Mental Health within Birmingham and Solihull Mental Health NHS Foundation Trust. $\mathrm{He}$ is also Professor of Psychiatry at the University of Birmingham, UK. Correspondence: Femi Oyebode, Department of Psychiatry, University of Birmingham, 25 Vincent Drive, Edgbaston, Birmingham B15 2FG, UK. Email: femi_oyebode@msn.com

\section{Copyright and usage} (C) The Royal College of Psychiatrists 2018 
BOX 1 Historical terms used to describe desire for amputation of a healthy limb or limbs

\section{Apotemnophilia}

From the Greek 'love to cut' or 'love for amputation'. Patients whose desire for selfamputation was felt to be related to sexual fantasy and arousal (Money 1977).

\section{Acrotomophilia}

Refers to the direction of the sexual fantasy felt in apotemnophilia towards other persons/ sexual partners who are amputees; also known as allo-apotemnophilia

Factitious disability disorder (FDD)

Used to describe two cases of patients seeking amputation whose motivations appeared to be driven by seeking care or attention for themselves (Bruno 1997).

Body dysmorphic disorder (BDD)

It was suggested that patients seeking selfamputation must be suffering from body dysmorphia, characterised by a persistent and delusional belief that some part of their body is deformed or excessively ugly (and therefore, by extension, there is motivation to seek removal of the offending body part) (Dyer 2000).

\section{Amputee identity disorder (AID)}

Offered as an alternative theory to those above, Fisher \& Smith (2000) wrote that this was an identity disorder rather than a paraphilia or body dysmorphia; the patient's primary motivation was a sense that they would become 'complete' once they became amputees.

Body integrity identity disorder (BIID)

The categorisation was broadened to describe a new identity disorder in which there is a persistent desire to acquire a physical disability and an associated sense of incompleteness at being able-bodied; there are similarities to gender identity disorder (First 2005, 2012).

\section{Xenomelia}

Literally 'foreign limb': this most recent term draws parallels with somatoparaphrenia (a neuropsychological syndrome that occurs mainly after right-hemisphere brain damage in which the patient denies ownership of a limb) (Sedda 2014).

\section{Conceptual history}

The concept of desire for amputation of a healthy limb is relatively new. Money et al (1977) wrote about the cases of two men who both sought an elective above-knee amputation. The authors had identified a paraphilia associated with amputees or self-amputeeism in a series of letters published in Penthouse magazine 5 years earlier. This syndrome was termed 'apotemnophilia' (amputation love) or 'acrotomophilia' (attraction to amputees) - see Box 1. The two men suffered from apotemnophilia, and their desire for self-amputation was described as 'an idée fixe rather than a delusion'.

Two decades later, Bruno (1997) proposed a different psychological concept for similar cases, that of factitious disability disorder (FDD). Bruno noted that internet forums and discussion groups were becoming more common and in such places people looking for sexual partners who were amputees were known as 'devotees'. Other categories were also established, known as 'pretenders' - people who pretended to have a disability through use of devices such as crutches or a wheelchair - and "wanabees' - people who themselves sought to acquire a disability, usually through limb amputation. Bruno proposed a psychological explanation for this disorder, in that FDD provided an opportunity for the person to be loved or attended to, and that the

disability could be their own or in someone else, real or pretended.

In September 1997, a Scottish surgeon, Dr Robert Smith, performed an elective above-knee amputation of a healthy limb on a patient from England. Two years later, in April 1999, he carried out a further above-knee amputation on a similar patient from Germany. Smith reported that both patients belonged to a small subgroup who desired elective amputation in order to feel they had only three limbs, not four (Dyer 2000). Sufferers of the condition that Smith described found their condition extremely distressing and disabling and often resorted to self-harm to get rid of the limb (Dyer 2000; Fisher 2000). Smith's employers, a National Health Service (NHS) trust, stopped any further procedures just before a third similar operation was to be carried out on an American. Since then there have been no further accounts of surgeons openly performing elective amputations of healthy limbs in the UK.

First (2005) reported a telephone interview study of 52 individuals who self-identified as having a desire for amputation. It was noted that none of the people included in the study were delusional or psychotic. In about three-quarters of the sample, their desire for amputation extended back to childhood or adolescence and was associated with distress, attempts at self-amputation or impairment in social or occupational functioning. In the majority (73\%) of those interviewed the primary goal of the amputation was to restore their perceived body identity, and not sexual arousal or gratification, therefore arguing against the idea that the amputation was driven by primary paraphilia (apotemnophilia). Hence, apotemnophilia was reconstrued as a disorder of body integrity and identity.

\section{Epidemiology}

Money's initial case reports (Money 1977) were both males and both identified as bisexual. Although separated, the first had been married and also had homosexual relationships and was able to recall a desire for amputation that began in early childhood and became a fixed desire from age 13 years. He recalled an injury to the affected leg when he was 2 years of age that left him unable to walk for 2 years. He was reported as having made a number of attempts to damage his leg either by introducing infection or using a tourniquet, although pain was in no way pleasurable for him. The second man recalled onset of desire for amputation from about the age of 11 years. He had attempted to secure an elective amputation a number of ways, but had not attempted selfamputation. 
A better indication of the characteristic age and gender distribution of BIID came from First's telephone survey (First 2005), which included 52 individuals, 47 of whom were male, 4 female and 1 male who was intersex at birth. This individual subsequently underwent male-to-female gender reassignment surgery. First noted a degree of potential referrer bias, as approximately two-thirds of participants were recruited to the study through the internet and the others through referral by other participants. There was a high incidence of homosexual males, possibly explained by the fact that one individual referred a further eight. Excluding those nine participants, $72 \%$ were heterosexual, 19\% homosexual and 9\% bisexual. All but two of the participants were white and most (90\%) had some education beyond high school. At the time of the study approximately $65 \%$ were unemployed, $7 \%$ were students and $23 \%$ had retired; the age range was 23-77 years.

A more recent survey of 54 individuals (Blom 2012) reported findings consistent with First's (2005): 80\% of the participants interviewed were male, and over $90 \%$ of white origin. Two-thirds were educated to university degree level and the age range of participants was 18-76 years. Respondents were sorted into two groups - amputation or paralysation (seeking severance of the spinal cord) depending on the specific desire for disability that they exhibited. Across both groups onset occurred in childhood or adolescence, at a mean age of 6-7 years.

Actual incidence of individuals who desire amputation is unknown but it is possible that the condition is not as rare as initially thought. Between 2000 and 2003 much media hype followed on from articles written about Dr Robert Smith and the elective amputations carried out in Scotland. Smith himself knew of other patients who desired similar operations (Fisher 2000; First 2012) and membership of online message board groups ranged from 1 to 2000 (Johnston 2002; First 2012). Accurate figures are difficult to determine from news articles and reports written about individuals who had sought or had surgical amputation as they have generally remained anonymous, probably owing to fears of stigma associated with wishing to acquire a disability. A review of the current internet presence of BIID suggests that some sites continue to exist (e.g. www.biid.org and www.overground.be) that, although primarily aimed at 'devotees' and 'wannabes', do contain information relating to BIID and personal stories and accounts of people who have successfully sought amputation. Blog posts by some individuals suggest that the larger groups have 'gone underground' because of the post-millennial explosion of the internet and the increased risk of susceptible individuals being targeted by people wishing to exploit their interest in finding a surgeon who would perform amputations. On the amputee-by-choice internet message board there are currently posts offering to put 'wannabe' amputees in touch with willing surgeons all over the world, but for a substantial fee (https://96528. activeboard.com). As can be expected, some responses to these posts are hopeful whereas others are sham. One message refers to a man known as the 'gatekeeper', who facilitates trips to Asia where amputations are performed on seemingly ordinary 'tourists' who present with 'symptoms of limb ischemia' and are then consented for limb-/life-saving surgery. However ordinary this approach to seeking treatment may seem, it is important to remember that unregulated surgical procedures are risky business and in 1998 US citizen Philip Bondy paid an unlicensed surgeon in Tijuana, Mexico, \$10000 for a healthy leg amputation and died of gangrene 2 days later in a San Diego hotel (https://caselaw. findlaw.com/ca-court-of-appeal/1300186.html).

\section{Pathogenesis}

There is currently no established or widely accepted understanding of the underlying pathogenesis of BIID. It was originally conceived as a paraphilia but there is growing evidence that this is not the whole story. Lawrence (2006) described a similarity with transsexualism because both conditions shared a number of features, including profound dissatisfaction with embodiment, sexual arousal from simulation of the sought-after status (pretending to be an amputee or transvestism) and attraction to persons with the same body type as the desired/target body type. This last feature is said to be prominent in non-homosexual male-to-female transsexuals (transsexual individuals who are not exclusively attracted to males) and is explained by a process termed erotic target location error. This process is thought to be present in some people with BIID. This hypothesis predicts that individuals who desire limb amputation will be noted also to be sexually attracted to amputees and in part this is determined by the fact that sexual 'aesthetic preference' for certain body morphology is dictated by the cortical representation of one's own body image (Ramachandran 2009). In a related but distinct thesis De Preester (2013) argued for a closer overlap between BIID and paraphilias, making the point that the sexual component is essential to the phenomenology of the condition. De Preester applied Merleau-Ponty's notion of sexual schema interacting with body image to produce the disorder that is manifest as BIID. The two hypotheses are complex and speculative in nature.

Attention has turned in recent times to probing and examining neurological aspects of BIID. There 
is reported evidence of heightened skin conductance response to pinprick below the line of the desired amputation in two individuals who had a longstanding wish for amputation of a limb (Brang 2008). This finding was interpreted as arising from a congenital dysfunction of the right superior parietal lobule and its connections to the insula. Earlier Ramachandran \& McGeoch (2007) had proposed that BIID was probably due to dysfunction of the right superior parietal lobule. This view was principally based on the reported preponderance of leftsided bias for the limb in question and the similarity between BIID and somatophrenia (a condition that occurs mainly after right hemisphere brain damage whereby patients deny ownership of their own $\mathrm{limb} / \mathrm{s}$, rejecting them as 'alien'). Furthermore, magnetoencephalography scans revealed that tactile stimulation of regions above and below the desired amputation line produced statistically reduced activation in the right superior parietal lobule (McGeoch 2011). This was interpreted as evidence of inadequate activation of the right superior parietal lobule, a brain area thought to integrate disparate sensory inputs into a coherent body image. The authors propose that BIID be renamed xenomelia to reflect the sense of estrangement of the affected limb. In a separate but similar investigation using functional magnetic resonance imaging (fMRI), it was shown that individuals with BIID showed heightened responsivity of a large somatosensory network, including the parietal cortex and right insula, regardless of whether the stimulated limb felt 'alien' or not (van Dijk 2013). Assessments of temporal order judgements of tactile stimulation proximal and distal to the desired amputation line revealed defective spatiotemporal integration specifically on the parts of the body that are undesired (Aoyama 2012). These findings seem to indicate that abnormal neural processes are likely to be at play in BIID (Sedda 2011, 2014), although it is probably premature to foreclose other possibilities

BOX 2 Clinical features of body integrity identity disorder

- Onset in childhood or early adolescence

- Delay in presentation to 30-50 years of age

- Predominantly affects males

- Increased prevalence of homosexual or bisexual orientation

- Association with gender identity disorder or other paraphilia

- Association with early exposure to an amputee during childhood

- Attempts at self-amputation
(Giummarra 2011). Finally, there is evidence of reduced cortical thickness in the superior parietal lobule and reduced cortical surface area in the primary and secondary somatosensory cortices in the inferior parietal lobule, as well as in the anterior insular cortex (Hilti 2013).

It has been suggested that the similarity between somatophrenia secondary to parietal lobe stroke means that BIID might be amenable to caloric vestibular stimulation (CVS) just as somatophrenia transiently responds to CVS (Ramachandran 2007). However, this has proven not to be the case (Lenggenhager 2014a). Nonetheless, there is evidence from the 'rubber foot illusion' (in which the individual feels illusory ownership of a fake foot after synchronous and asynchronous stroking of a visible rubber foot and their hidden foot) that individuals with BIID experienced an increase in the vividness of the illusion for the undesired foot compared with healthy controls (Lenggenhager 2014b). This finding was interpreted as demonstrating a weakened representation of the affected body part and it strengthens the possibility that multisensory stimulation might provide therapeutic benefit.

\section{Clinical features}

The clinical features described below and summarised in Box 2 are drawn from the following studies: First (2005), Blom et al (2012), Bou Khalil \& Richa (2012) and First \& Fisher (2012). There is a persistent desire to acquire a significant disability, the onset of which occurs during childhood or early adolescence, that is, between 8 and 12 years of age. The primary motivation is to feel 'whole' or 'complete' and serves to rectify the individual's image of their true identity (as an amputee or paraplegic or with a significant disability), without which a sense of intense discomfort or inappropriateness would persist. There is impairment in social or occupational functioning and/or frequent attempts at self-amputation resulting from significant psychological distress. Despite the early age at onset, presentation most commonly occurs between 30 and 50 years of age, and often following a failed self-amputation attempt. This delay is thought to be due to perceived stigma or a fear of adverse judgement from relatives or medical professionals.

In addition, there is a predominance of males with BIID. There appears to be an important sexual component, in that the majority of respondents to the survey by First (2005) reported sexual attraction to other amputees and around half reported that sexual arousal was a secondary reason for desiring amputation. Many people with BIID also engage in 'pretending' behaviour such as wrapping up their limbs or using aids and adaptions (Blom 2012). 
This is often compared with cross-dressing in transsexualism.

There is no evidence that individuals with BIID have a positive family history of psychiatric disorder. Bou Khalil \& Richa (2012) suggest that there may be an association with DSM-IV cluster B personality disorders, but this view is based on individual case reports rather than large-scale surveys.

\section{Treatment}

Individuals with BIID are most likely to be offered a psychological intervention such as cognitive-behavioural therapy or psychotherapy as a management option or pharmacological treatment with a selective serotonin reuptake inhibitor or other antidepressant medication. First (2005) reported that $65 \%$ of respondents in his survey had been in psychotherapy at some point but almost half of those had never disclosed their BIID to their therapist. Although not curative, these treatments may well alleviate distress temporarily and would suggest that there is an association with mood symptoms that are probably experienced secondary to the psychological distress associated with BIID.

\section{Ethics}

Medical ethical considerations are considered using the four-principles approach developed by Beauchamp \& Childress (2013), namely respect for autonomy, non-maleficence, beneficence and justice. This approach has become very influential, especially in the English-speaking world. In this approach, it is recognised that these principles are not absolute and often require balancing in order to come to clinical decisions. The framework allows for a reasoned approach to decision-making in situations in which moral dilemmas exist. The principles are general guides that allow for judgement, taking into account the specificities and contextual aspects of each case, in reaching decisions. Our aim here is not to provide a detailed ethical or conceptual analysis of BIID but to summarise the headline considerations currently evident in the literature.

BIID came to widespread public attention in the UK in 2000, following a BBC television documentary about the amputation of healthy limbs in two individuals by Dr Robert Smith, a surgeon at Falkirk and District Royal Infirmary, Scotland (Horizon 2000). Dr Smith later reported that follow-up of three patients who had had elective amputations between 1997 and 2000 revealed that none required further treatment (Fisher 2000).

The emotions aroused in thinking about ethical aspects of the surgical treatment of BIID are well illustrated in the following statement, which was published shortly after the amputation of the healthy limbs of the two individuals was first revealed in the press: 'Removing people's physically healthy limbs because they have a psychological condition is not only a dangerous precedent to set but also is a case where the few must suffer to safeguard the majority. These amputations could eventually lead to the acceptance of self-harm without significant attempts to address the underlying psychological causes of self-loathing' (Beckford-Ball 2000). There appear to be at least two concerns here, namely that the surgical removal of healthy limbs for the treatment of a psychological condition cannot be right and that accepting this approach to the management of psychological distress may be the thin edge of the wedge that could lead to even more unacceptable demands for surgical interventions that are merely a means of achieving selfharm. There are a number of implicit assumptions in Beckford-Ball's paper, many of which hint at moral disapproval and not merely professional disagreement over the nature of disease and what counts as appropriate treatment. In other words, BIID raises fundamental moral issues regarding its status as a medical condition, the appropriateness of surgical intervention that causes harm by amputating a healthy limb and the possibility of harmful consequences not merely for the individual patient but for society as a whole.

\section{Non-maleficence}

In this section we examine whether surgical treatment for BIID is in breach of the ethical principle of nonmaleficence. There is an already well-established principle in medicine to 'at least do no harm', this being the maxim primum non nocere. This obligation to do no harm and its related but distinct obligation to use treatment to benefit the sick are foundational obligations in medicine. The conceptual problem is how to determine the nature of harm and this is a truly complex matter. Beauchamp \& Childress (2013) make the case that serious harm involves setbacks to physical and psychological interests, including such physical harms as pain, disability and death. Therefore, on the face of it, surgical amputation of a healthy limb self-evidently causes harm as it causes manifest disability. This issue of the amputation of a healthy limb seems to be at the root of much of the negative emotional response against surgical treatment for BIID.

Johnston \& Elliot (2002) make a strong case against the amputation of healthy limbs in BIID. They acknowledge that the problem here is not simply that of the surgical removal of a healthy limb, since there are at least three other situations 
in which there is elective removal of a healthy body part: cosmetic surgery that is justified by the patient's own aesthetic preference; living-donor organ transplantation; and gender reassignment surgery. The pivotal argument seems to be that a surgeon who performs elective amputations of healthy limbs may be at risk of a medical malpractice suit because the procedure is not yet considered by a responsible body of medical opinion to be an appropriate and effective treatment of a medical condition. Perhaps more importantly, they make the case that a surgeon in this situation might be liable to a charge of criminal assault. They base their argument on $R$ v Brown [1994], in which a group of men who had videotaped themselves performing consensual sadomasochistic activities, which included branding, burning, hitting of the genitals, whipping, caning, biting and stinging with nettles, were found guilty under the Offences Against the Person Act 1861 (governing England, Wales and Northern Ireland). The House of Lords, by a majority ruling, held that the presence of consent is not a defence against a charge of assault that has caused actual bodily harm. The dissenting minority opinion in this case drew a distinction between actual bodily harm and grievous bodily harm, specifically that consent would not even in their view be a defence against a charge of grievous bodily harm. Johnston $\&$ Elliot continue, 'Whatever the roots of the desire for amputation may be, the boundaries of the condition are flexible and overlapping with other social phenomena'. They conclude, 'It is not yet clear that the desire for amputation is properly seen as a medical disorder, let alone that amputation of the limb is the appropriate response'.

To summarise, the argument seems to be that surgical intervention in BIID is unethical because the provenance of the condition is yet to be fully established. In other words, healthy organs can be surgically removed if and only if there is consensus about the underlying nature of the condition under consideration. And, in Johnston \& Elliot's view, consent of the patient to the surgery will be of no assistance to the surgeon should litigation arise.

\section{Beneficence and autonomy}

We now examine whether there are moral arguments in support of surgical intervention for BIID. Bayne \& Levy (2005), unlike Johnston \& Elliot above, argue in favour of a limited role for elective amputation for BIID. In essence, their argument is that (a) elective amputation minimises harm by securing proper medical treatment under appropriate supervision rather than exposing patients with BIID to risky unsupervised surgery, (b) individuals who are acting autonomously and who have capacity ought to have their preferences and desires given due weight, (c) treatment - that is, elective amputation - is likely to secure relief from suffering that cannot be obtained by less radical means, and (d) the relief of suffering is therefore worth the cost of surgery. Bayne $\&$ Levy are aware that, whatever the arguments for elective amputation of a healthy limb in BIID are, many people will continue to find the idea objectionable or repugnant.

The case that is being made by Bayne $\&$ Levy derives from the principles of beneficence and autonomy. There is a conceptual distinction between nonmaleficence and beneficence. It is usually argued that beneficence is promoted by action that prevents harm, removes harm or promotes good. The point Bayne $\mathcal{E}$ Levy seem to be making is that BIID is a condition that causes harm, namely mental suffering, and that doctors have a duty to relieve harm and suffering and are under an obligation to act with beneficence in mind. We reiterate that patients with BIID suffer from psychological distress and often seek to secure amputations either through self-harm or from unorthodox and suspect agents. Beauchamp \& Childress (2013: p. 207) specify the conditions that need to be satisfied for an obligation of beneficence to exist between two people (A and B) and these are the following: (a) A is at significant risk of loss or damage to life or health; (b) B's actions are needed to prevent this loss or damage; (c) B's actions have a high probability of preventing the loss or damage; (d) B's actions would not present significant risks, costs or burdens to $\mathrm{B}$; and (e) the benefit that $\mathrm{A}$ can expect to gain outweighs any harms, costs or burdens that B is likely to incur.

A structured approach such as that proposed by Beauchamp \& Childress helps us to disentangle the complex of possible responses to BIID. It is true that some people with BIID are at significant risk of loss or damage and it can be argued that B's action, namely surgery, is required to prevent the risks to A described above. It is also true that B's action of surgical intervention stands a high chance of preventing the presumed loss but paradoxically it can only achieve this by causing loss and disability. There are considerable risks to $\mathrm{B}$, including the likelihood of litigation as well as loss of professional reputation for performing surgery that is yet to be accepted as appropriate in this set of circumstances. The surgical amputation of a healthy limb to cause disability remains stubbornly problematic.

The other arm of Bayne \& Levy's argument is that autonomous individuals have a compelling right to expect that their reasonable requests are responded to with due consideration. It is not that an individual who has capacity has an unquestionable right to require another person to cause harm to them but 
that the request must be given due weight. The principle of respect for autonomy is an overarching and deeply important moral principle in Western society. It presupposes that individuals are autonomous actors who act freely in accordance with a self-chosen life goal. As Beauchamp \& Childress put it 'To respect an autonomous agent is, at a minimum, to acknowledge that person's right to hold views, to make choices, and to take actions based on personal values and beliefs' (Beauchamp $\&$ Childress 2013: p. 106). It matters not that the choice may not accord with the action preferred by another person or that the action may even be deemed wrong and ill judged by others. Such is the dominance of the principle of autonomy in the healthcare arena that is assumed that patients are acting autonomously until proven otherwise. Issues of consent, of the capacity to give consent and of refusal of treatment all flow from our concepts of autonomy. But equally, autonomy only has prima facie standing and can be overridden by competing moral considerations. And there are limits to what an autonomous agent can ask other autonomous actors to do for or to them. We have shown above that there is a line of reasoning that argues that doctors who intentionally cause harm to an autonomous and consenting person may still be in legal jeopardy.

The view put forward by Bayne $\&$ Levy above is not without its critics. In particular, Patrone (2009) argues among other things that wider social costs such as disabled parking places, social support and adaptations to buildings and homes complicate matters. In other words, that the socalled treatment has wider implications, including the just distribution of resources for the benefit of others within a community, is of relevance here. This further argument is that due regard must be paid to the ways in which medical interventions influence resource use. This view is already incorporated in the list of conditions that Beauchamp $\&$ Childress say must be satisfied for an obligation of beneficence to exist between two people - see (d) and (e) above. However, these conditions refer only to costs or burdens that B bears, whereas here Patrone refers to societal costs and burdens. The concern that Patrone expresses is best described as distributive justice, a term that refers to the fair, equitable and appropriate distribution of resources in society determined by justified norms that structure the terms of social cooperation. This principle is important in a healthcare system such as the NHS, which has limited and finite resources. Resources that are allocated for one procedure, for example surgical amputation of a healthy limb, will by definition affect the funding of some other procedure for another condition. It is a truism that issues of distributive justice arise and become salient under conditions of scarcity and competition.

Our own view is that BIID is likely to be a neuropsychiatric disorder reflecting abnormalities of body image and body representation due to dysfunction in the right superior parietal lobule. This view suggests that BIID is not simply a personal preference but a rare and distressing medical condition. On this view, it could be argued that the treatments available, including elective amputation, are designed to manage a neuropsychiatric disorder rather than a socially desirable preference. In any case it can hardly be plausibly argued that seeking a disability is a socially desirable outcome. In other words, BIID can be conceived as a disorder with demonstrable underlying abnormality that causes suffering. It is therefore appropriate to relieve suffering with the treatments that are available. This is not to argue that surgical amputation or spinal transection ought to be first-line treatments but simply to state that BIID is a condition that, despite our natural moral feelings of repugnance or disapproval, merits our compassion and concern and that the treatments that are available, including surgical amputation of a healthy limb, ought to be given serious consideration. There is no doubt, however, that in due course non-surgical, and thereby less radical, treatment may become available. The possibility that multimodal sensory stimulation might produce therapeutic benefit has to be kept in mind. BIID is a complex condition that requires sensitivity and awareness of the distress that it causes and also an openness about what the appropriate and morally correct position towards it ought to be.

\section{Conclusions}

BIID is a rare but intriguing condition that is yet to be fully understood. The conceptual framework for investigating it has evolved over the past 40 years, from a description that privileged the paraphilic aspects of the clinical features to more recent accounts that favour neuropsychological and neuropsychiatric processes that are thought to be manifestations of dysfunction in the right superior parietal lobule. There is undoubted moral discomfort, if not repugnance, at the notion of elective amputation of a healthy limb but this may be partially modified by a realisation that this unusual phenomenon is a reflection of underlying pathophysiology. There is, in our view, no logical difference between the conceptual status of BIID and transsexualism. Hence, given that individuals with transsexualism are offered gender reassignment surgery it seems to us that individuals with BIID ought at least to be considered for treatment, including elective amputation in some cases. It may be that the need for this radical 
MCO answers and controversial form of treatment would soon be obviated by novel treatments involving multimodal sensory stimulation. There is no doubt though that BIID is a morally challenging condition and that the use of surgical amputation or transection of the spine as treatment is deeply troubling.

\section{References}

Aoyama A, Krummenacher P, Palla A, et al (2012) Impaired spatial-temporal integration of touch in xenomelia (body integrity identity disorder). Spatial Cognition \& Computation, 12: 96-110.

American Psychiatric Association (2013) Diagnostic and Statistical Manual of Mental Disorders (5th edn) (DSM-5). APA.

Bayne T, Levy N (2005) Amputees by choice: body integrity identity disorder and the ethics of amputation. Journal of Applied Philosophy, 22: 75-86.

Beauchamp TL, Childress JF (2013) Principles of Biomedical Ethics (7th edn). Oxford University Press.

Beckford-Ball J (2000) The amputation of healthy limbs is not an option. British Journal of Nursing, 9(4): 188

Blom RM, Hennekam RC, Denys D (2012) Body integrity identity disorder. PloS One, 7(4): e34702.

Bou Khalil R, Richa S (2012) Apotemnophilia or body integrity identity disorder: a case report review. International Journal of Lower Extremity Wounds, 11: 313-9.

Brang D, McGeoch PD, Ramachandran VS (2008) Apotemnophilia: a neurological disorder. Neuroreport, 19: 1305-6.

Bruno RL (1997) Devotees, pretenders and wannabes: two cases of factitious disability disorder. Sexuality and Disability, 15: 243-60.

De Preester H (2013) Merleau-Ponty's sexual schema and the sexual component of body integrity identity disorder. Medicine, Health Care, and Philosophy, 16: 171-84.

Dyer C (2000) Surgeon amputated healthy legs. BMJ, 320: 332

First MB (2005) Desire for amputation of a limb: paraphilia, psychosis, or a new type of identity disorder. Psychological Medicine, 35: 919-28.

First MB, Fisher CE (2012) Body integrity identity disorder: the persistent desire to acquire a physical disability. Psychopathology, 45: 3-14.

Fisher K, Smith R (2000) More work is needed to explain why patients ask for amputation of healthy limbs. BMJ, 320: 1147.

Giummarra MJ, Bradshaw JL, Nicholls ME, et al (2011) Body integrity identity disorder: deranged body processing, right fronto-parietal dys- function, and phenomenological experience of body incongruity. Neuropsychology Review, 21: 320-33.

Hilti LM, Hänggi J, Vitacco DA, et al (2013) The desire for healthy limb amputation: structural brain correlates and clinical features of xenomelia. Brain, 136: 318-29.

Horizon (2000) Complete obsession. BBC 2, 17 February (https://www.bbc. co.uk/science/horizon/1999/obsession_script.shtml).

Johnston J, Elliott C (2002) Healthy limb amputation: ethical and legal aspects. Clinical Medicine, 2: 431-5.

Lawrence AA (2006) Clinical and theoretical parallels between desire for limb amputation and gender identity disorder. Archives of Sexual Behavior, 35: 263-78.

Lenggenhager B, Hilti L, Palia A, et al (2014a) Vestibular stimulation does not diminish the desire for amputation. Cortex, 54: 210-2.

Lenggenhager B, Hilti L, Brugger P (2014b) Disturbed body integrity and the 'rubber foot illusion'. Neuropsychology, 29: 205-11.

McGeoch PD, Brang D, Song T, et al (2011) Xenomelia: a new right parietal lobe syndrome. Journal of Neurology, Neurosurgery, and Psychiatry, 82: $1314-9$.

Money J, Jobaris R, Furth G (1977) Apotemnophilia: two cases of self-demand amputation as a paraphilia. Journal of Sex Research, 13: $115-25$.

Patrone D (2009) Disfigured anatomies and imperfect analogies: body integrity identity disorder and the supposed right to selfdemanded amputation of healthy body parts. Journal of Medical Ethics, 35: 541-5.

\section{$R$ v Brown [1994] 1 AC 212.}

Ramachandran VS, McGeoch P (2007) Can vestibular caloric stimulation be used to treat apotemnophilia? Medical Hypotheses, 69: 250-2

Ramachandran VS, Brang D, McGeoch PD, et al (2009) Sexual and food preference in apotemnophilia and anorexia: interactions between 'beliefs' and 'needs' regulated by two-way connections between body image and limbic structures. Perception, 38: 775-7.

Sedda A (2011) Body integrity identity disorder: from a psychological to a neurological syndrome. Neuropsychology Review, 21: 334-6.

Sedda A, Bottini G (2014) Apotemnophilia, body integrity identity disorder or xenomelia? Psychiatric and neurologic etiologies face each other. Neuropsychiatric Disease and Treatment, 10: 1255-65.

van Dijk MT, van Wingen GA, van Lammeren A, et al (2013) Neural basis of limb ownership in individuals with body integrity identity disorder. PloS One, 8(8): e72212.

World Health Organization (2018) ICD-11: International Classification of Diseases 11th Revision. WHO. 
MCOs

Select the single best option for each question stem

1 Giving consideration to the ethical arguments for the role of elective surgical amputation as a treatment for body integrity identity disorder (BIID), which of the following is thought to be incorrect?

a relief of suffering is worth the cost of surgery

b elective amputation minimises harm by preventing patients with BIID being exposed to risky, unsupervised surgery or making attempts at selfamputation

c it is patients' right to have their preferences or desires given true weight and consideration, provided they are acting autonomously and there is no impairment of decision-making capacity

$d$ there are no current elective surgical practices that condone the removal of healthy body parts

$\mathrm{e}$ it is a treatment that may have an impact on the just distribution of resources.
2 The clinical features of body integrity identity disorder include all of the following, except:

a predominantly affects male

b predominantly affects the right lower limb (leading to requests for right above-knee amputations)

c previous attempts at self-amputation, or selfamputation behaviour

d delay in onset of presentation (usually to between 30 and 50 years of age)

e associated with significant psychological distress or impairment in functioning.

3 Body integrity identity disorder is defined as:

a a type of body dysmorphia in which the individual believes that the affected limb is ugly or deformed

b the persistent desire to acquire a physical disability such as amputation, paraplegia or other severe disability such as blindness

c the desire for self-amputation in order to fulfil sexual gratification or sexual preference

d attraction to amputees

e the desire to aquire a disability in order to receive care.
4 Non-maleficence is an ethical principle that: a relies on the person's capacity to make decisions for themselves

b involves the requirement that the person be willing to accept harmful care

c is an example of the just distribution of resources

d is based on the professional-patient relationship

e flows from the ethical principle primum non nocere.

5 The principle of autonomy:

a demands that the patient's request for particular treatment must always be met

b involves treating the patient's wishes with respect

c is an absolute moral principle in medicine

$\mathrm{d}$ is the same as the capacity to give consent to treatment

$\mathrm{e}$ is synonymous with the principle of beneficence. 